\title{
Synchronization and bistability of qubit coupled to a driven dissipative oscillator
}

\author{
O.V.Zhirov ${ }^{1}$ and D.L.Shepelyansky ${ }^{2}$ \\ ${ }^{1}$ Budker Institute of Nuclear Physics, 630090 Novosibirsk, Russia \\ ${ }^{2}$ Laboratoire de Physique Théorique, UMR 5152 du CNRS, Université Toulouse III, 31062 Toulouse, France
}

(Dated: October 10, 2007)

\begin{abstract}
We study numerically the behavior of qubit coupled to a quantum dissipative driven oscillator (resonator). Above a critical coupling strength the qubit rotations become synchronized with the oscillator phase. In the synchronized regime, at certain parameters, the qubit exhibits tunneling between two orientations with a macroscopic change of number of photons in the resonator. The life times in these metastable states can be enormously large. The synchronization leads to a drastic change of qubit radiation spectrum with appearance of narrow lines corresponding to recently observed single artificial-atom lasing [O. Astafiev et al. Nature 449, 588 (2007)].

PACS numbers: 74.50.+r, 42.50.Lc, 03.65.Ta
\end{abstract}

In physics there are not so many simple quantum problems which are exactly solvable [1]. Two of them are monochromatically driven two-level atom (spin-half or qubit) [2] and quantum oscillator (unitary or dissipative) $[3,4]$. One atom weakly coupled to a field in a resonator is known as the Jaynes-Cummings model which is also integrable $[5,6,7]$. At strong coupling the dynamics may become nontrivial with the emergence of classical $[8,9]$ and quantum chaos [10] but in this case one should have many atoms which may absorb many photons. For one atom even with a strong coupling to a quantum photonic field the dynamics is still relatively simple due to a total energy balance [11].

This old problem of a two-level atom coupled to photons regained recently a significant interest due to appearance of long living superconducting qubits [12] which can be strongly coupled to a microwave resonator $[13,14,15]$. There are also other possibilities of superconducting qubit coupling to a quantum oscillator [16, 17]. The oscillator can be realized as a tank circuit tuned to the Rabi frequency [16] or as a current-biased dc SQUID [17] allowing efficient energy exchange with a qubit. Possibilities of qubit coupling to a cooled nanomechanical resonator are actively discussed $[18,19]$ and coupling between micro-mechanical cantilever and atomic spin found impressive experimental implementations (see [20] and Refs. therein). However, the most intriguing way seems to be the coupling with a microwave resonator where a lasing has been realized recently with 6 - 30 photons pumped into the resonator [15]. Contrary to recent interesting theoretical studies [20,21,22] where pumping is applied to a spin or qubit we concentrate here on the case where a monochromatic pumping is applied to a dissipative oscillator (resonator). Such a dissipative oscillator can be also viewed as a semiclassical detector which performs monitoring of a qubit. This continuous type of measurements is now actively discussed for superconducting qubits and other solid-state devices [23]. The continuous measurement of a superconducting qubit is realized in [16].
The Hamiltonian of our model reads

$$
\hat{H}=\hbar \omega_{0} \hat{n}-\hbar \Omega \sigma_{x} / 2+g \hbar \omega_{0}\left(\hat{a}+\hat{a}^{\dagger}\right) \sigma_{z}+f \cos \omega t\left(\hat{a}+\hat{a}^{\dagger}\right)
$$

where $g$ is a dimensionless coupling constant, the driving force amplitude and frequency are $f=\hbar \lambda \sqrt{n_{p}}$ and $\omega$, the oscillator frequency is $\omega_{0}$ and $\hbar \Omega$ is the qubit energy spacing. As in [22] we choose a qubit coupling via $\sigma_{z}$. We assume that the qubit life time is enormously long and that its dynamics is perturbed only by the coupling with the driven dissipative oscillator. The dissipation rate of oscillator is $\lambda$ and we assume the quality factor to be $Q=\omega_{0} / \lambda \sim 100$. The evolution of the whole system is described by the master equation for the density matrix $\hat{\rho}$ which has the standard form [4]:

$$
\dot{\hat{\rho}}=-\frac{i}{\hbar}[\hat{H}, \hat{\rho}]+\lambda\left(\hat{a} \hat{\rho} \hat{a}^{\dagger}-\frac{1}{2} \hat{a}^{\dagger} \hat{\rho} \hat{a}-\frac{1}{2} \hat{\rho} \hat{a}^{\dagger} \hat{a}\right)
$$

The numerical simulations are done by direct solution of time evolution of $\hat{\rho}$ expanded in a finite basis of oscillator states $n$, by the state diffusion method [24] and by the method of Quantum Trajectories (QT) [25]. We ensured that these methods give the same results but the majority of data is obtained with quantum trajectories which we found to be more suitable for massive simulations. In addition the QT have an advantage of providing a pictorial illustration of individual experimental runs. The numerical details are the same as in $[26,27]$ and we use here up to $n=70$ oscillator states which give good numerical convergence. Our results show that a coupling of two simple integrable models gives a nontrivial interesting behavior.

A typical example of QT is shown in Fig. 1. It shows two main properties of the evolution: the oscillator spends a very long time at some average level $\langle n\rangle=n_{-}$and then jumps to another significantly different value $n_{+}$. At the same time the polarization vector of qubit $\vec{\xi}$ defined as $\vec{\xi}=\operatorname{Tr}(\hat{\rho} \vec{\sigma})$ also changes its orientation direction with a clear change of sign of $\xi_{x}$ from $\xi_{x}>0$ to $\xi_{x}<0$. The time averaged values of $\xi_{y, z}$ 


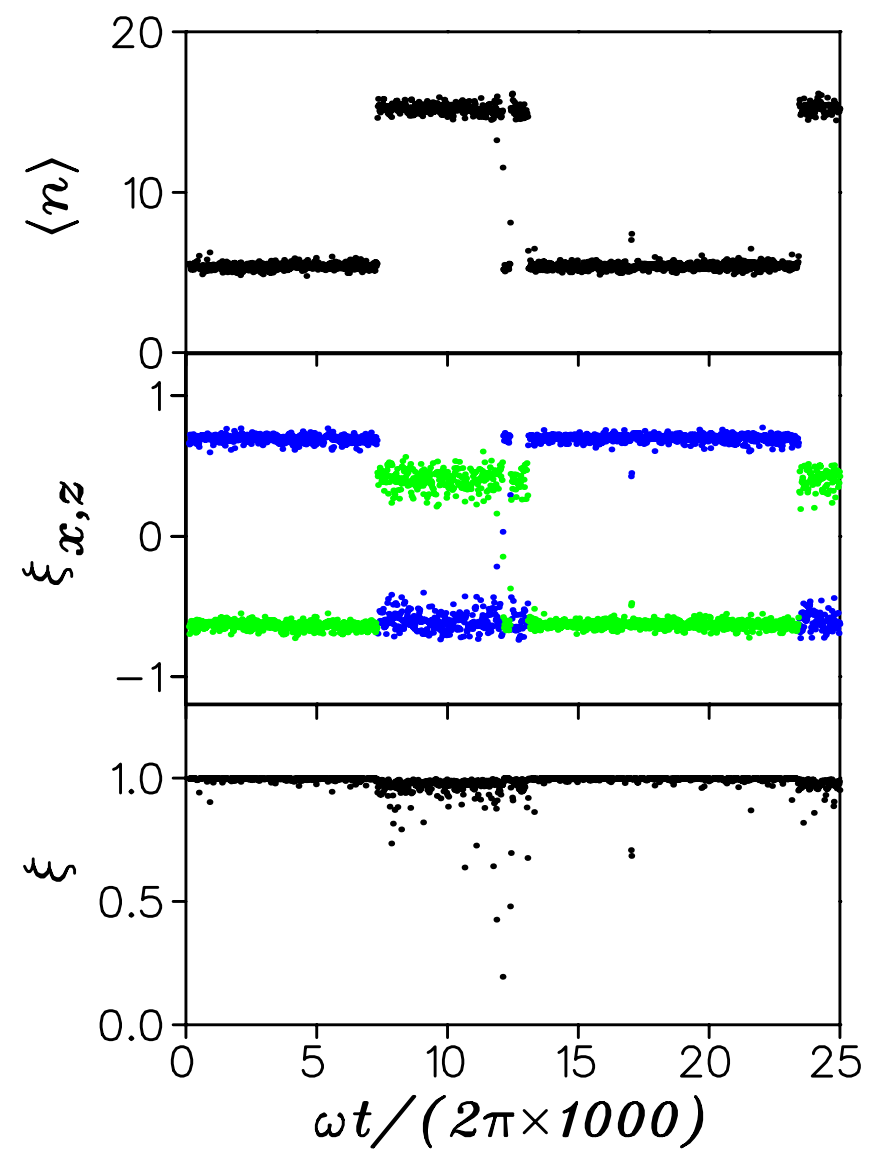

FIG. 1: (color online) Bistability of qubit coupled to a driven oscillator with jumps between two metastable states. Top panel shows average oscillator level number $\langle n\rangle$ as a function of time $t$ at stroboscopic integer values $\omega t / 2 \pi$; middle panel shows the qubit polarization vector components $\xi_{x}$ (blue/black) and $\xi_{z}$ (green/gray) at the same moments of time; the bottom panel shows the degree of qubit polarization $\xi$. Here the system parameters are $\lambda / \omega_{0}=0.02, \omega / \omega_{0}=1.01$, $\Omega / \omega_{0}=1.2, f=\hbar \lambda \sqrt{n_{p}}, n_{p}=20$ and $g=0.04$.

are zero but when they are taken at stroboscopic integer moments $\omega t / 2 \pi$ they also show transitions between two metastable states. The transition time is approximately $t_{m} \sim 1 / \lambda$ being rather small compare to the life time in a metastable state. Inside such a state the degree of qubit polarization $\xi=|\vec{\xi}|$ is very close to unity showing that the qubit remains mainly in a pure state. The drops of $\xi$ appear only during transitions between metastable states. Special checks show that an inversion of $\xi_{x}$ by an additional pulse (e.g. from $\xi_{x}>0$ to $\xi_{x}<0$ ) produces a transition of oscillator to a corresponding state (from $n_{-}$to $n_{+}$) after time $t_{m} \sim 1 / \lambda$. Thus we have here an interesting situation when a quantum flip of qubit produces a marcoscopic change of a state of detector (oscillator) which is continuously coupled to a qubit (we checked that even larger variation $n_{ \pm} \sim n_{p}$ is possible by taking $n_{p}=40$ ). In addition to that inside a metastable state the coupling induces a synchronization of qubit ro-
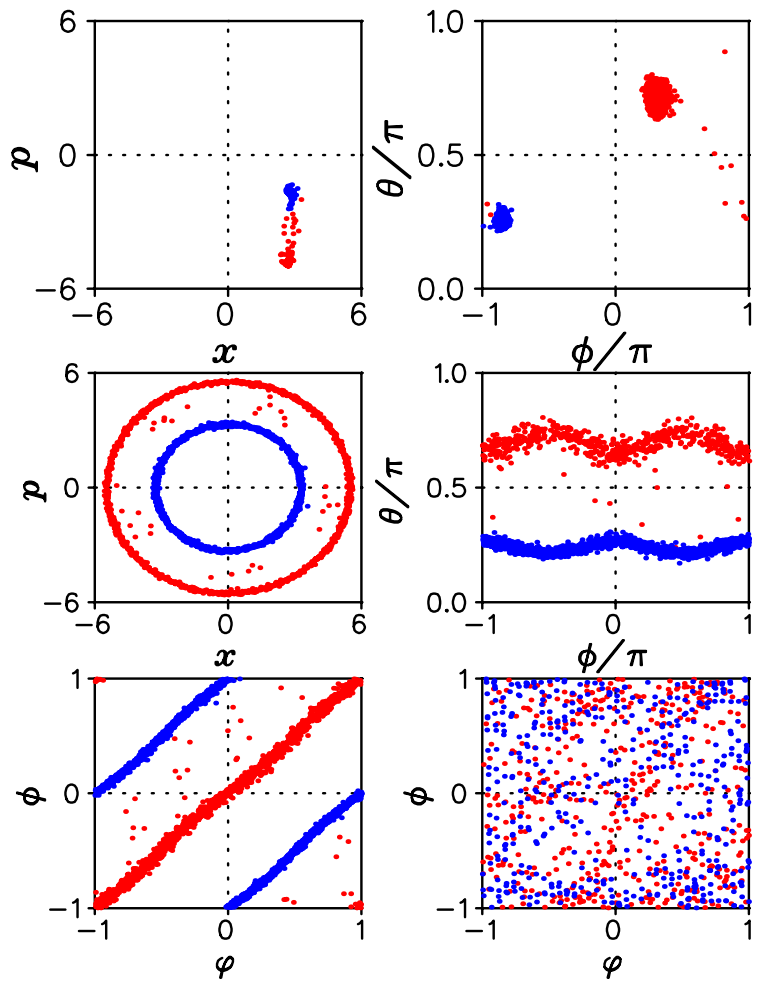

FIG. 2: (color online) Top panels: the Poincaré section taken at integer values of $\omega t / 2 \pi$ for oscillator with $x=$ $\left\langle\left(\hat{a}+\hat{a}^{\dagger}\right) / \sqrt{2}\right\rangle, p=\left\langle\left(\hat{a}-\hat{a}^{\dagger}\right) / \sqrt{2} i\right\rangle$ (left) and for qubit polarization with polarization angles $(\theta, \phi)$ defined in text (right). Middle panels: the same quantities shown at irrational moments of $\omega t / 2 \pi$. Bottom panels: the qubit polarization phase $\phi$ vs. oscillator phase $\varphi(p / x=-\tan \varphi)$ at time moments as in middle panels for $g=0.04$ (left) and $g=0.004$ (right). Other parameters and the time interval are as in Fig. 1. The color of points is blue/black for $\xi_{x}>0$ and red/gray for $\xi_{x}<0$.

tation phase with the oscillator phase which in its turn is fixed by the phase of driving field. The synchronization is a universal phenomenon for classical dissipative systems [28]. It is known that it also exists for dissipative quantum systems at small effective values of $\hbar$ [27]. However, here we have a new unusual case of qubit synchronization when a semiclassical system produces synchronization of a pure quantum two-level system.

The phenomenon of qubit synchronization is illustrated in a more clear way in Fig. 2. The top panels taken at integer values $\omega t / 2 \pi$ show the existence of two fixed points in the phase space of oscillator (left) and qubit (right) coupled by quantum tunneling (the angles are determined as $\xi_{x}=\xi \cos \theta, \xi_{y}=\xi \sin \theta \sin \phi, \xi_{z}=$ $\xi \sin \theta \cos \phi$ ). A certain scattering of points in a spot of finite size should be attributed to quantum fluctuations. But the fact that on enormously long time (Fig. 1) the spot size remains finite clearly implies that the oscillator phase $\varphi$ is locked with the driving phase $\omega t$ inducing the qubit synchronization with $\varphi$ and $\omega t$. The plot at $t$ val- 

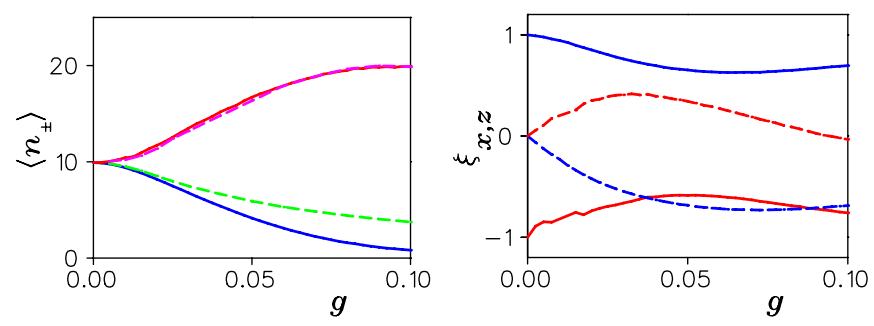

FIG. 3: (color online) Right panel: dependence of average qubit polarization components $\xi_{x}$ and $\xi_{z}$ (full and dashed curves) on $g$, averaging is done over stroboscopic times (see Fig.1) in the interval $100 \leq \omega t / 2 \pi \leq 2 \times 10^{4}$; color is fixed by the sign of $\xi_{x}$ averaged over 10 periods (red/gray for $\xi_{x}<0$, blue/black for $\xi_{x}>0$; this choice fixes also the color on right panel). Left panel: dependence of average level of oscillator in two metastable states on coupling strength $g$, the color is fixed by the sign of $\xi_{x}$ on right panel that gives red/gray for large $n_{+}$and blue/black for small $n_{-}$; average is done over the quantum state and stroboscopic times as in the left panel; dashed curves show theory dependence (see text)). Two QT are used with initial value $\xi_{x}= \pm 1$. All parameters are as in Fig. 1 except $g$.

ues incommensurate with $2 \pi / \omega$ (middle panels) shows that in time the oscillator performs circle rotations in $(p, x)$ plane with frequency $\omega$ while qubit polarization rotates around $x$-axis with the same frequency. Quantum tunneling gives transitions between two metastable states. The synchronization of qubit phase $\phi$ with oscillator phase $\varphi$ is clearly seen in bottom left panel where points form two lines corresponding to two metastable states. This synchronization disappears below a certain critical coupling $g_{c}$ where the points become scattered over the whole plane (panel bottom right). It is clear that quantum fluctuations destroy synchronization for $g<g_{c}$. Our data give $g_{c} \simeq 0.008$ for parameters of Fig. 1 .

The variation of bistable states with coupling strength $g$ is shown in Fig. 3. The difference between $n_{+}$and $n_{-}$grows with $g$. It is striking that $n_{-}$may become close to zero. The direction of qubit polarization also changes in a smooth but nontrivial way. It is also important to note that according to our data the dispersion of oscillator wave function in metastable states is compatible with the dispersion of a coherent state with $n_{ \pm}$. This corresponds to a wave packet collapse induced by dissipation (see $[26,27]$ and Refs. therein). The dependence of $n_{ \pm}$on driving frequency $\omega$ is shown in Fig. 4. A symmetric double peak structure is evident: for $\omega>\omega_{0}$ the metastable state with $\xi_{x}<0$ has maximal $n$ value while for $\omega<\omega_{0}$ the state with maximal $n$ has $\xi_{x}>0$ (note color interchange). The peak width is approximately equal to the dissipation rate $\lambda$. With the increase of $g$ their form becomes asymmetric indicating importance of nonlinear effects. The splitting of peaks grows approximately linearly with $g$ (inset at Fig. 4) and reminds the vacuum Rabi splitting effect [6]. The shift

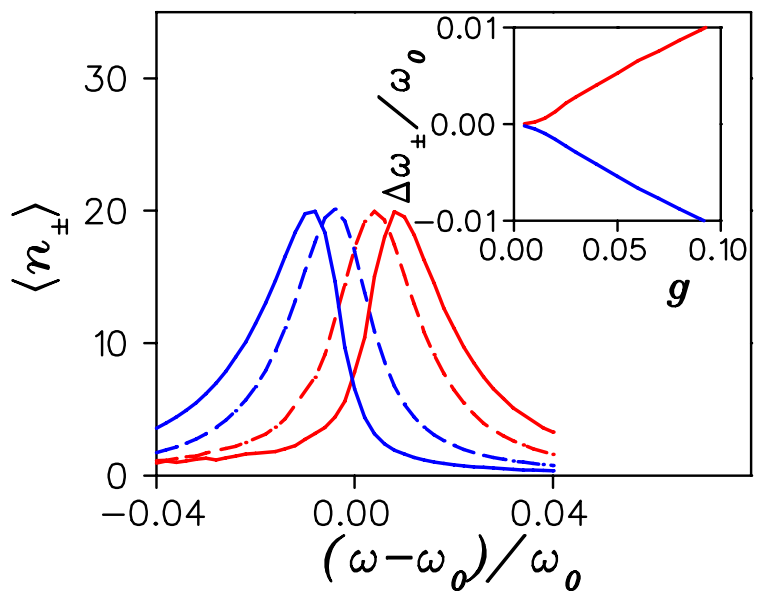

FIG. 4: (color online) Dependence of average level $n_{ \pm}$of oscillator in two metastable states on the driving frequency $\omega$ (average and color choice are the same as in right panel of Fig. 3); coupling is $g=0.04$ and $g=0.08$ (dashed and full curves). Inset shows the variation of position of maximum at $\omega=\omega_{ \pm}$with coupling strength $g, \Delta \omega_{ \pm}=\omega_{ \pm}-\omega_{0}$. Other parameters are as in Fig. 1.

$\Delta \omega_{ \pm}$explains two states $n_{ \pm}$of driven oscillator well described by $\left.n_{ \pm}=n_{p} \lambda^{2} /\left(4\left(\omega-\omega_{0}-\Delta \omega_{ \pm}\right)^{2}+\lambda^{2}\right)\right]$ (see dashed curves in Fig. 3 left traced with numerical values of $\Delta \omega_{ \pm}$from Fig. 4 inset). To estimate $\Delta \omega_{ \pm}$we note that the frequency of effective Rabi oscillations between quasidegenerate levels is $\Omega_{R} \approx g \omega_{0} \sqrt{n_{ \pm}+1}[5,7]$ that gives $\Delta \omega_{ \pm} \approx d \Omega_{R} / d n \approx \pm g \omega_{0} / 2 \sqrt{n_{ \pm}+1}$ in a good agreement with data of Fig. 4 for moderate $g$.

The properties of two metastable states are analyzed in Fig. 5. The number of transitions $N_{f}$ between these states has a pronounced peak at $\Omega \approx 1.1 \omega_{0}$ that approximately corresponds to a resonance condition $\Omega-\omega \approx$ $2 g \omega_{0}$. For $\Omega<1.08 \omega_{0}$ there is an abrupt drop of $N_{f}$ and bistability becomes irregular disappearing for certain $\Omega$, but the synchronization still remains. Quite interestingly, the data show that for $\Omega>1.1 \omega_{0}$ the life times of each state are rather different and enormously large, generally $\tau_{-}>\tau_{+} \gg \omega_{0} / \lambda$.

The spectrum of qubit radiation $S(\nu)$ in presence of phase noise in $\phi$ is shown in Fig. 6. It confirms the main features discussed above: for $\Omega / \omega_{0}=1.2$ the growth of driving power $n_{p}$ induces the synchronization of qubit with radiation suppression at qubit frequency $\Omega=1.2 \omega_{0}$ and appearance of narrow line with lasing at $\nu=\omega$. For $\Omega=\omega_{0}$ the radiation spectrum $S(\nu)$ at $n_{p}<1$ has two broad peaks at $\nu=\omega \pm g \omega_{0}$ corresponding to the vacuum Rabi splitting [6] (narrow line from driving source at $\nu=$ $\omega$ is also visible in this case). At strong driving $n_{p}>1$ the synchronization takes place with appearance of one lasing line at $\nu=\omega$. For both values of $\Omega$ the transition to synchronization/lasing takes place at $n_{p}>n_{p l} \approx 2$. The spectrum $S(\nu)$ in Fig. 6 has close similarities with the spectrum observed recently in a single artificial-atom 


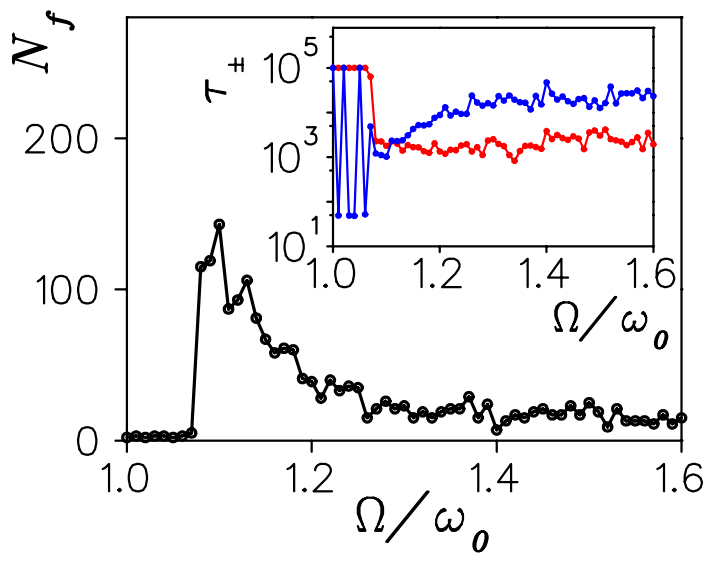

FIG. 5: (color online) Dependence of number of transitions $N_{f}$ between metastable states on rescaled qubit frequency $\Omega / \omega_{0}$ for parameters of Fig. $1 ; N_{f}$ are computed along 2 QT of length $10^{5}$ driving periods. Inset shows life time dependence on $\Omega / \omega_{0}$ for two metastable states $\left(\tau_{+}\right.$for red/gray, $\tau_{-}$for blue/black, $\tau_{ \pm}$are given in number of driving periods; color choice is as in Figs. 2,3).
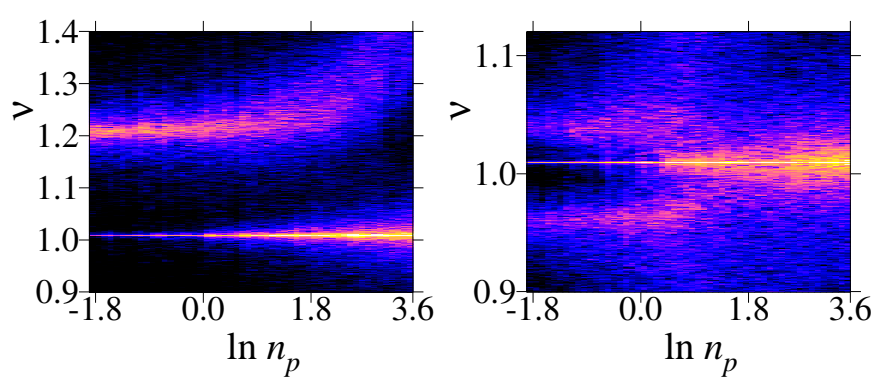

FIG. 6: (color online) Spectral density $S(\nu)$ of qubit radiation $\xi_{z}(t)$ as function of driving power $n_{p}$ in presence of phase noise in $\phi$ with diffusion rate $\eta=0.004 \omega_{0}$. Left: $\Omega / \omega_{0}=$ 1.2 ; right: $\Omega / \omega_{0}=1$. Other parameters are as in Fig. 1 . Color/grayness shows $S(\nu)$ in logarithmic scale (white/black for maximal/zero), $\nu$ is given in units of $\omega_{0}$.

lasing [15] which appears at a similar threshold $n_{p l} \approx 1$. A shift related to splitting $\omega_{ \pm} \approx g \omega_{0} / 2 \sqrt{n}$ is also seen experimentally. However, the vacuum Rabi splitting is not visible in Fig.3c of [15]. Exact comparison requires much more extended numerical simulations since in [15] $Q \approx 10^{4}$ while we have $Q \sim 100$.

In conclusion our numerical studies show a nontrivial behavior of a rather simple model given by Eqs. $(1,2)$. It is characterized by bistability and synchronization of qubit induced by its coupling to a quantum driven dissipative oscillator. As for the vacuum Rabi splitting [6] it is important that the oscillator is quantum since the effect is absent for a classical dissipative oscillator with commuting $a, a^{\dagger}(1)$. A better analytical description and understanding of the behavior discussed requires further studies. Especially interesting is the analysis of long life times $\tau_{ \pm}$in metastable states related to a macroscopic quantum tunneling.
Preparing the paper to submission we became aware of the preprint [29] where a similar model is studied but synchronization is not discussed there.

This work is supported by the EC project EuroSQIP and RAS joint scientific program "Fundamental problems of nonlinear dynamics" (for OVZ).

[1] P.M. Morse and H. Feshbach, Methods of Theoretical Physics, McGraw-Hill Sci. (1953).

[2] L.D. Landau and E.M. Lifshits, Quantum mechanics, Nauka, Moskva (1989).

[3] A. Perelomov, Generalized Coherent States and Their Applications, Springer, Berlin (1986).

[4] U. Weiss, Dissipative quantum mechanics. World Sci., Singapore (1999).

[5] E.T. Jaynes and F.W. Cummings, Proc. IEEE 51, 89 (1963).

[6] J.J. Sanchez-Mondragon, N.B. Narozhny, and J.H. Eberly, Phys. Rev. Lett. 51, 550 (1983).

[7] M.O. Scully and M.S. Zubairy, Quantum optics, Cambridge Univ. Press, Cambridge (1997).

[8] P.I. Belobrov, G.M. Zaslavsky, and G.K. Tartakovsky, Zh. Eksp. Teor. Fiz. 71, 1799 (1976) [Sov. Phys. JETP 44, 945 (1976)].

[9] J.R. Ackerhalt, P.W. Milonni, and M.-L. Shin, Phys. Rep. 128, 205 (1985).

[10] R. Graham and M. Höhnerbach, Phys. Rev. Lett. 57, 1378 (1986).

[11] R. Graham and M. Höhnerbach, Z. Phys. B 57, 233 (1984); M. Kuś, Phys. Rev. Lett. 54, 1343 (1985).

[12] D. Vion et al., Science 296, 886 (2002).

[13] A. Wallraff et al., Nature 431, 162 (2004).

[14] A.A. Houck et al., Nature 449, 328 (2007).

[15] O. Astafiev et al., Nature 449, 588 (2007).

[16] E. Il'ichev et al., Phys. Rev. Lett. 91, 097906 (2003).

[17] J. Claudon et al., Phys. Rev. Lett. 93, 187003 (2004).

[18] A.N. Cleland and M.R. Geller, Phys. Rev. Lett. 93, 070501 (2004).

[19] M. Poggio et al., Phys. Rev. Lett. 99, 017201 (2007).

[20] G.P. Berman al., IEEE Trans. Nanotech. 4, 14 (2005).

[21] D.A. Rodrigues, J. Imbers, and A.D. Armour, Phys. Rev. Lett. 98, 067204 (2007).

[22] J. Hauss et al., preprint arXiv:cond-mat/0701041v5 (2007).

[23] A.N. Korotkov, Phys. Rev. B 60, 5737 (1999); ibid. 67, 235408 (2003); A.N. Korotkov and D.V. Averin, ibid. 64, 165310 (2001).

[24] N. Gisin and I.Percival, J.Phys. A 25, 5677 (1992); ibid. 26, 2233 (1993); ibid. 26, 2245 (1993).

[25] T.A. Brun, I.C. Percival, and R. Schack, J. Phys. A 29, 2077 (1996); T.A. Brun, Am. J. Phys. 70, 719 (2002).

[26] G. Carlo, G. Benenti, and D.L. Shepelyansky, Phys. Rev. Lett. 95, 164101 (2005).

[27] O.V. Zhirov and D.L. Shepelyansky, Eur. Phys. J. D 38, 375 (2006).

[28] A. Pikovsky, M. Rosenblum, and J. Kurths, Synchronization: A Universal Concept in Nonlinear Sciences, Cambridge Univ. Press, Cambridge (2001).

[29] J. Gambetta et al., preprint arXiv:0709.4264 (2007). 